\title{
Biofunctionalisation of gallium arsenide with neutravidin
}

\author{
Bárbara Santos Gomes, ${ }^{*}, \dagger$ David J. Morgan, ${ }^{\ddagger}$ Wolfgang Langbein, ${ }^{\top}$ Paola Borri, ${ }^{\dagger}$ and Francesco Masia ${ }^{\dagger}$ \\ $\dagger$ School of Biosciences, Cardiff University, Cardiff CF10 3AX, United Kingdom \\ $\ddagger$ Cardiff Catalysis Institute, School of Chemistry, Cardiff University, Cardiff CF10 3AT, United Kingdom \\ 【School of Physics \&5 Astronomy, Cardiff University, Cardiff CF24 3AA, United Kingdom \\ E-mail: SantosGomesB@cardiff.ac.uk
}

\begin{abstract}
Gallium arsenide (GaAs) is a promising candidate as a platform for optical biosensing devices due to its enabling optoelectronic properties. However, the biofunctionalisation of the GaAs surface has not received much attention compared to gold, carbon and silicon surfaces. Here we report a study presenting a physicochemical surface characterisation of the GaAs surface along the functionalisation with a high-affinity bioconjugation pair widely explored in the life sciences - biotin and neutravidin. Combined X-ray photoelectron spectroscopy (XPS), wettability measurements and spectroscopic ellipsometry were used for a reliable characterisation of the surface functionalisation process. The results suggest that a film with a thickness lower than $10 \mathrm{~nm}$ was formed, with a neutravidin to biotin ratio of $1: 25$ on the GaAs surface. Reduction of non-specific binding of the protein to the surface was achieved by optimising the protein buffer and rinsing steps. This study shows the feasibility of using GaAs as a platform for specific biomolecular recognition, paving the way to a new generation of optoelectronic biosensors.
\end{abstract}

\section{Introduction}

Gallium arsenide (GaAs) is a compound semiconductor material with unique optical and electrical properties, currently used for a wide range of applications, including biosensing devices. ${ }^{1-3}$ In contrast to silicon, whose indirect band gap hinders the integration of light sources in a lab-onchip design, direct band-gap semiconductors such as GaAs are suited materials for high-speed high-efficiency optoelectronic devices including light sources such as light emitting diodes or lasers. These materials have the potential to provide miniaturised biosensors integrated with CMOS technology, when combined with appropriately engineered biocomponents. When developing such biosensors, one crucial step is the functionalisation of the sensing platform with biological recognition elements (so-called bioreceptors). ${ }^{4,5}$ While a variety of systematic studies have been reported for the biofunctionalisation of gold (both flat $\mathrm{Au}$ surfaces ${ }^{6}$ and $\mathrm{Au}$ nanoparticles ${ }^{7}$ ), silicon, ${ }^{8}$ and carbon, ${ }^{9}$ relatively little work has been done exploring the biofunctionalisation of GaAs and the optimisation of such functionalisation strategies, including reduction of non-specific binding. Investigation of passive adsorption of proteins, rather than biospecific interaction of the immobilised ligand with its biological counterpart, is particularly important when functionalising surfaces for biosensing applications. ${ }^{10}$
Nanoscale films with a wide variety of functional groups can be formed by simple self-assembly on surfaces, ${ }^{11}$ which then allow further functionalisation with bioreceptors. ${ }^{12}$ Taking advantage of the highly covalent $\mathrm{As}-\mathrm{S}$ bond, ${ }^{13}$ alkanethiolate self-assembled monolayers (SAMs) have previously been formed on GaAs surfaces. ${ }^{14}$ These SAMs have been created mostly for GaAs surface electronic and chemical passivation ${ }^{15-17}$ but also for further functionalisation with peptides, ${ }^{18}$ bacteria, ${ }^{19}$ virus, ${ }^{20}$ and proteins. ${ }^{21}$ While several studies provided a thorough characterisation of alkanethiolate SAMs on GaAs, ${ }^{22-27}$ further functionalisation stages with biomolecules have not been systematically investigated.

This study comprises a detailed physicochemical surface characterisation for GaAs functionalisation with a system widely exploited in the life sciences, biotin/avidin. ${ }^{6,8,28-30}$ The popularity of this system stems from (i) the strong and rapid interaction between the biotin and avidin, (ii) the high binding affinity and specificity, (iii) the ability to withstand extreme conditions (in terms of $\mathrm{pH}$, temperature, organic solvents and denaturing reagents) and (iv) the multivalent properties of avidin, with its capacity to bind 4 biotin molecules.

In this study, the GaAs surface is firstly functionalised with oligo(ethyl glycol)-containing (OEG-) alkanethiolate films. Several studies have revealed that the presence of OEG or PEG moieties on the surface can improve the resistance to non-specific binding. ${ }^{31-34}$ However, this ability to resist protein adsorption is not definite and can vary for example according to the molecular conformation of OEGterminated SAMs formed on those surfaces. ${ }^{35}$ SAM formation is followed by covalent attachment of a biotin-based molecule. Subsequent bioconjugation of biotin with its high binding affinity partner - neutravidin - is then promoted. Neutravidin is a deglycosylated version of avidin, which exhibits a lower isoelectronic point and lower nonspecific physical adsorption than avidin, while still retaining the properties mentioned above.

Here we use combined X-ray photoelectron spectroscopy (XPS), ellipsometry and wettability measurements to characterise the surface at different stages of functionalisation and to investigate the ability of this dual-component SAM to resist non-specific binding of neutravidin. 


\section{Materials and Methods}

\section{Materials}

GaAs wafers (prime grade, VGF growth, single crystal, orientation $(100) \pm 0.1^{\circ}$, undoped, single side polished, 3 " diameter, $625 \pm 25 \mu \mathrm{m}$ thickness) were purchased from Wafer Technology. PBS (phosphate-buffered saline 1x, $\mathrm{pH} 7.4), \quad \mathrm{BupH}^{\mathrm{TM}} \mathrm{MES}$ (2-(N-Morpholino)ethanesulfonic acid) buffered saline pack, amine-PEG2-biotin and NeutrAvidin ${ }^{\mathrm{TM}}$ biotin-binding protein (neutravidin) were purchased from Fisher Scientific. Sulfo-NHS (Nhydroxysulfosuccinimide sodium salt) was purchased from Tokyo Chemical Industry. Unless otherwise specified, chemicals were purchased from Sigma-Aldrich. Solvents of ACS grade were used.

\section{Methods}

\section{Sample preparation}

GaAs wafers were cleaved into $(5 \times 5) \mathrm{mm}^{2}$ pieces using a diamond scribe. The pieces were cleaned with subsequent ultrasonic baths ( $5 \mathrm{~min}$ each) in acetone, ethanol and isopropanol, respectively, and dried with $\mathrm{N}_{2}$ flow. Immediately before functionalisation with thiols, the GaAs oxide layer was removed by an acidic bath $(2 \mathrm{~min}$ in $\mathrm{HCl} 1 \mathrm{M})$ followed by rinsing with water and ethanol. For SAM formation, the samples were incubated in a freshly prepared thiol solution for $24 \mathrm{~h}$, under gentle agitation using an orbital shaker. A mixture of ethanol and water $(2: 1)$ with $10 \%$ of acetic acid was used as solvent for the two thiols (carboxyl-OEG-thiol, CAS 866889-02-3, and hydroxyl-OEG-thiol, CAS 13072741-2), which were in solution at 1:1 molar ratio, at a final concentration of $50 \mathrm{\mu M}$ for each thiol. The use of a mixture of water and ethanol as solvent for thiol solutions has been reported to form superior quality mixed alkanethiolate SAMs, when compared to the use of ethanol only. ${ }^{25}$ After SAM formation, the samples were rinsed with the same solvent mixture and dried under $\mathrm{N}_{2}$ flow. For the attachment of the biotin molecule, via carbodiimide chemistry, the samples were incubated for $30 \mathrm{~min}$ in a freshly prepared mixture of N-hydroxysulfosuccinimide (NHS) and N-(3Dimethylaminopropyl)-N'-ethylcarbodiimide hydrochloride (EDC) in MES 0.1 M, pH 4.7. The final concentration in solution was $50 \mathrm{mM}$ of sulfo-NHS, and $0.2 \mathrm{M}$ of EDC. The samples were thoroughly rinsed with the same buffer (MES 0.1 M, pH 4.7), to remove excess products and byproducts. Afterwards, the samples were rinsed with PBS to raise the surface $\mathrm{pH}$ and then incubated with biotin solution $(50 \mu \mathrm{g} / \mathrm{mL}$ in PBS) for $2 \mathrm{~h}$. After incubation with biotin, the samples were rinsed with flowing PBS followed by an ultrasonic bath in PBS for $10 \mathrm{~min}$. The unreacted NHS-ester groups were blocked by incubating the samples in alkaline hydroxylamine solution (1 M, pH 8.5), followed by rinsing with flowing PBS and drying under $\mathrm{N}_{2}$ flow. For neutravidin attachment, the samples were incubated for $2 \mathrm{~h}$ in $50 \mathrm{\mu g} / \mathrm{mL}$ solution of neutravidin diluted in either PBS or PBST (i.e. PBS with $0.05 \%$ TWEEN ${ }^{\circledR} 20, \mathrm{pH} 6.3$ ), and then rinsed in a sequence of PBST and PBS baths with agitation (1 min in PBST followed by $4 \mathrm{~min}$ in PBS, changing the buffer every minute; then repeating the whole procedure 3 times, for a total rinsing time of $20 \mathrm{~min}$ ) and drying under $\mathrm{N}_{2}$ flow. Table 1 summarises the samples at different stages of the functionalisation analysed in this work.
Table 1: Overview of the samples investigated. $M$ denotes monolayer, $\mathrm{B}$ biotin, and $\mathrm{N}$ neutravidin.

\begin{tabular}{|c|l|c|c|}
\hline ID & Stage & $\begin{array}{c}\text { Neutravidin } \\
\text { buffer }\end{array}$ & $\begin{array}{c}\text { Washing } \\
\text { buffer }\end{array}$ \\
\hline \hline$\# 1$ & Bare GaAs & - & - \\
\hline$\# 2$ & Oxidised GaAs & - & - \\
\hline$\# 3$ & GaAs-M & - & - \\
\hline$\# 4$ & GaAs-M-B & - & - \\
\hline$\# 5$ & GaAs-M-B-N & PBS & PBS \\
\hline$\# 6$ & GaAs-M-B-N & PBS & PBS/PBST \\
\hline$\# 7$ & GaAs-M-B-N & PBST & PBS/PBST \\
\hline$\# 8$ & GaAs-M-N & PBS & PBS \\
\hline$\# 9$ & GaAs-M-N & PBS & PBS/PBST \\
\hline$\# 10$ & GaAs-M-N & PBST & PBS/PBST \\
\hline
\end{tabular}

\section{Contact Angle}

Static contact angle measurements were performed by placing a $2 \mu l$ water droplet on the sample surface and capturing an image (with a digital camera Nikon Coolpix L120) from the top (Fig. S3). A reference pattern was included in the picture to calibrate the camera pixel and measure the droplet diameter using ImageJ. Finally, the contact angle $(\theta)$ was calculated by numerically solving Eq.(1) which expresses the dependence of $\theta$ on the wetted radius $(r)$ and the droplet volume $(V)$, with the assumption the shape of the droplet is a segment of a sphere. ${ }^{36}$

$$
\frac{3 V}{\pi r^{3}}=\frac{2-3 \cos (\theta)+\cos ^{3}(\theta)}{\sin ^{3}(\theta)}
$$

The error of the contact angle measurement $\left(\sigma_{\theta}\right)$ was calculated from Eq.(2), which accounts for the error propagation using the functional dependence in Eq.(1), with the error of the volume $\left(\sigma_{V}\right)$ being taken as the maximum random error of the pipette $(0.013 \mathrm{\mu l})$ and the error of the radius $\left(\sigma_{r}\right)$ estimated as $0.05 \mathrm{~mm}$.

$$
\sigma_{\theta}=\frac{1+2 \cos (\theta)+\cos ^{2}(\theta)}{\pi r^{3}} \sqrt{\sigma_{V}^{2}+\left(3 V \frac{\sigma_{r}}{r}\right)^{2}}
$$

\section{Spectroscopic Ellipsometry}

A Woollam M200D ellipsometer was used for acquisition of the ellipsometric parameters ( $\Psi$ and $\Delta$, in degrees) as a function of the wavelength (spectral range of 200 to $1000 \mathrm{~nm}$ ). $\Delta$ represents the phase shift of the light reflected from the surface polarized in the plane of incidence relative to the one polarized perpendicular to that plane, and tan $(\Psi)$ represents the ratio of their amplitudes. Measurements were undertaken at 5 angles of incidence: $65^{\circ}, 68^{\circ}, 71^{\circ}, 74^{\circ}$ and $77^{\circ}$, with an acquisition time of $1 \mathrm{~s}$ and a beam diameter of approx. $4 \mathrm{~mm}$. For thickness calculation, CompleteEASE software (version 5.13) was used and a model consisting of one or more Cauchy transparent layers on GaAs was applied to a wavelength range of 300 to $850 \mathrm{~nm}$, simultaneously to $\Delta$ and $\Psi$ data for the five angles of incidence. The fit range has been selected to discard the absorption of the organic layer (for wavelengths shorter than $300 \mathrm{~nm}$ ) and to avoid multiple reflections arising from the bottom surface once the GaAs becomes transparent at wavelengths longer than $850 \mathrm{~nm}$. The model comprised a GaAs (100) substrate (optical constants uploaded from the software library, shown in Fig. S4) covered by one or more layers (more details on 
the number of layers used for each sample are shown in SI, Table S1) described by a 2-parameter Cauchy dispersion model, where the dependence of the refractive index $n$ on the wavelength lambda is given by

$$
n(\lambda)=A+\frac{B}{\lambda^{2}},
$$

and the extinction coefficient $k$ is set to 0 . An additional term proportional to $\lambda^{-4}$ can be added to Eq.(3), but it was found that it did not improve the fit. $A=1.5$ and $B=0.01 \mathrm{\mu m}^{2}$ were used as initial guesses, and the fit used the constrain $B \geq 0$. The initial guess value for thickness was $2.8 \mathrm{~nm}$. When more than one layer was added to the model, only the parameters for the top layer were varied by the fit while the properties of the underlying layers were fixed using the results from the previous steps in the functionalisation. For samples where neutravidin was present, the fitting improved significantly (more than $10 \%$ improvement in the mean squared error, MSE) if $k$ was described by an Urbach absorption tail

$$
k(\lambda)=k_{0} e^{\alpha \lambda}
$$

where $k_{0}$ and $\alpha$ are fit parameters that determine the shape of the extinction coefficient dispersion. The MSE was calculated by the software and used for evaluation of the goodness of the fitting.

\section{X-ray Photoelectron Spectroscopy (XPS)}

XPS measurements were performed using a Thermo Scientific $\mathrm{K}$-alpha ${ }^{+}$system with a monochromatic $\mathrm{Al} \mathrm{K \alpha}$ source $(1486.6 \mathrm{eV})$. Charge compensation (at low power, to avoid degradation) was applied to prevent sample charging. An analysis area of $(400 \times 400) \mathrm{\mu m}^{2}$ and a take-off angle of $90^{\circ}$ was used. High resolution spectra were acquired at a pass energy of $40 \mathrm{eV}$ (20 scans, dwell time $0.05 \mathrm{~s}$ ). For the bare GaAs measurements (\# 1), argon clusters (cluster size of 150 atoms at $6 \mathrm{keV}$, giving an energy of $40 \mathrm{eV}$ per atom) were used to remove oxides from the GaAs surface. CasaXPS software (version 2.3.23rev1.1N) was used for peak fitting using a least-squares method. For each selected region of a scan, linear or 2 parameter Tougaard (so called U2 Tougaard) backgrounds were applied and peak models were created using LA (Lorentzian asymmetric) lineshapes. ${ }^{37,38}$ Elemental relative sensitivity factors (RSF) used for the correction of elemental/component areas were selected from the Scofield library. All the scans were calibrated to the $\mathrm{C} 1$ s component at $284.8 \mathrm{eV}$. For the calculation of the C 1s spectral components, the position (i.e. binding energy) of each component was fixed and the full width at half maximum (FWHM) was constrained to be the same for all the components. The area for the $\mathrm{N}$ 1s component was calculated by applying position and FWHM constrains (allowing to move $\pm 0.2 \mathrm{eV}$ ) to the overlapping peaks coming from the substrate. These were assessed from the GaAs-M scan and also verified by a high resolution scan of GaAs acquired just after in situ oxide layer removal with argon clusters (not shown).

\section{Results}

We initiate the functionalisation by creating a dualcomponent alkanethiolate SAM on GaAs, using the components hydroxyl-OEG-thiol and carboxyl-OEG-thiol (molecular structures shown in Fig. S1). The carboxyl groups are used for the immobilisation of a biotin- $\mathrm{NH}_{2}$ (see Fig. S1c) molecule via carbodiimide crosslinking (see Fig. S2), while the hydroxyl-OEG-thiol is used as a spacer, allowing control over the density of the immobilised biomolecule, and also providing more rigidity to the SAM through the $\mathrm{C}_{11} \mathrm{H}_{23}$ alkyl chain. Following the attachment of biotin to the carboxyl groups, via stable amide bonds, the surface is exposed to neutravidin, with consequent immobilisation of the protein on the surface. A schematic representation of the functionalisation strategy is presented in Fig. 1.

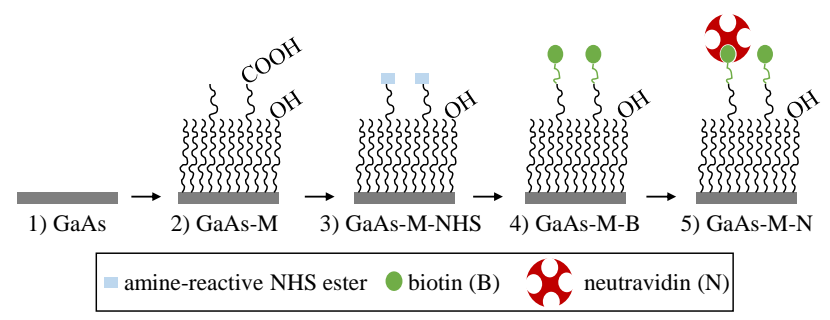

Figure 1: Schematic representation of GaAs functionalisation strategy, showing dual-component alkanethiolate monolayer on GaAs with carboxyl and hydroxyl functional groups (2), intermediate amine-reactive N-hydroxysulfosuccinimide (NHS) ester (3), biotin immobilisation (4), and neutravidin immobilisation (5).

\section{Wettability}

Water contact angle measurements were used for investigation of changes in wettability induced by hydrophilic groups (such as carboxyl groups and the aminoacids located on the protein outer surface) immobilised on the GaAs surface. Static water contact angle values for different stages of GaAs functionalisation are shown in Fig. 2. Gallium and arsenic oxides that are formed on the GaAs surface as a result of air exposure ${ }^{39}$ were removed by means of an acidic bath, immediately before SAM formation. The oxide-free GaAs (\#1, see Table 1) surface is hydrophobic, ${ }^{40}$ exhibiting a contact angle value higher than $90^{\circ}$. After SAM formation (\#3), the measured contact angle was about $67^{\circ}$. The dualcomponent thiol-based SAM on GaAs was formed by incubation in a solution of equal molar fractions of carboxylOEG-thiol and hydroxyl-OEG-thiol. Despite the presence of hydrophilic terminal groups (carboxyl and hydroxyl), the measured contact angle is not below $40^{\circ}$, as commonly reported for carboxyl- and hydroxyl-terminated alkanethiolate SAMs. ${ }^{41,42}$ We attribute this to a contribution from a) the protonation of carboxylate groups ${ }^{43}$ and also to b) the ethylene glycol units. Indeed, it has been previously shown, for gold and glass substrates, that OEG-containing SAMs result in a more hydrophobic and flexible surface than the pure alkanethiolate SAMs. ${ }^{44,45}$ Following biotin attachment $(\# 4)$, the contact angle value increased slightly $\left(76^{\circ}\right)$ due to less carboxyl groups present on the surface. After protein attachment, the surface became more hydrophilic due to aminoacids being preferentially located on the outer surface of the protein. ${ }^{46,47}$ Varying the protein dilution buffer - PBS (pH 7.4, stage \#5) vs. PBST (PBS with $0.05 \%$ TWEEN ${ }^{\circledR} 20$, pH 6.3 , stage \#7) - resulted in different contact angle values. Diluting the protein in PBS resulted in a lower contact angle $\left(35^{\circ}\right)$, when compared to PBST $\left(48^{\circ}\right)$, suggesting different amounts of protein present on these surfaces. To confirm if the additional protein coverage observed 
in stage \#5 could be a result of neutravidin non-specific physical adsorption onto the SAM via electrostatic interactions, rather than high-affinity biospecific conjugation of neutravidin to biotin, further investigations were performed and are discussed below.

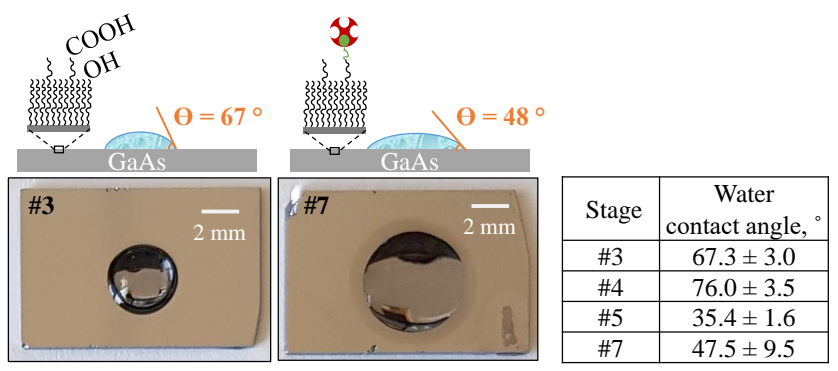

Figure 2: Images (top view) of water droplets on GaAs samples at different stages of functionalisation, with a schematic representation of the drop profile and corresponding contact angle. $10 \mu \mathrm{L}$ droplets were used for visualisation purposes. An increase in hydrophilicity was observed after protein attachment. The table presents static water contact angles measured for different functionalisation stages and protein dilution buffer. Sample stages are given in Table 1. The values are the average of measurements on triplicate samples, with the errors representing the standard deviation. The largest measurement error in the contact angle measurement was calculated to be of $0.91^{\circ}$, smaller than the observed standard deviation values.

\section{Spectroscopic ellipsometry}

We investigated the changes in the optical properties of the surface by spectroscopic ellipsometry measurements. This technique measures the change in the polarization of the light upon reflection from the sample surface. Such change is represented by the amplitude $(\tan (\Psi))$ and phase $(\Delta)$ of the ratio of the complex reflectance of the $\mathrm{p}$ (in the plane of incidence) and s field (perpendicular to $\mathrm{p}$ ). Film properties such as thickness and refractive index can be calculated from $\Psi$ and $\Delta$ using an optical model to fit the data. The spectra of $\Delta$ and $\Psi$ for different stages of functionalisation of GaAs are presented in Fig. 3. Bare GaAs (\#1) could not be measured as the surface oxidises instantly upon contact with air. Both parameters changed after immobilisation of biotin (\#4) and then after incubation with neutravidin (\#7), as expected, confirming changes of the surface. As the functionalisation progressed, we observed a decrease in $\Delta$ which can be interpreted as an increase in the film thickness, as suggested by fitting the spectra with a multi-layer model. In particular, we observed a decrease in the visibility of the peak around $900 \mathrm{~nm}$, which is a signature of the substrate. Thickness values fitted for these samples are shown in Table 2. Detailed information on the Cauchy model used for each stage, and additional calculated parameters, can be found in SI (Table $\mathrm{S} 1$ ). The thickness value obtained for the $\mathrm{GaAs}-\mathrm{OH} / \mathrm{COOH}$ SAM (\#3), $2.3 \mathrm{~nm}$, suggests the formation of a monolayer and is in accordance with a theoretical length of $2.6 \mathrm{~nm}$ and $3.3 \mathrm{~nm}$ for the fully extended hydroxyl-OEG-thiol and carboxyl-OEG-thiol molecules, respectively. This length was calculated using Chem3D software and is based on the distance measured from the $\mathrm{S}$ atom to the farthest $\mathrm{C}$ atom in the fully extended molecule. As the functionalisation proceeds, after GaAs-OH/COOH SAM formation, the samples are expected to be composed of more heterogeneous layers, therefore requiring more complex ellipsometric models to fit the data. In addition, for thin films (i.e. with thickness lower than $10 \mathrm{~nm}$ ) this technique is not as sensitive to changes in the refractive index as it is to variations in the thickness. ${ }^{48}$ Nevertheless, an increase in thickness of the films as the functionalisation proceeds is clearly observed.

For optical biosensing exploiting the evanescent wave of light at the GaAs/water interface, it is important that the film thickness is smaller than the decay length of the optical field, which is in the order of $100 \mathrm{~nm}$ for light in the near infrared. The measured total thickness of the film does not exceed $10 \mathrm{~nm}$, confirming the suitability of the reported functionalisation strategy.
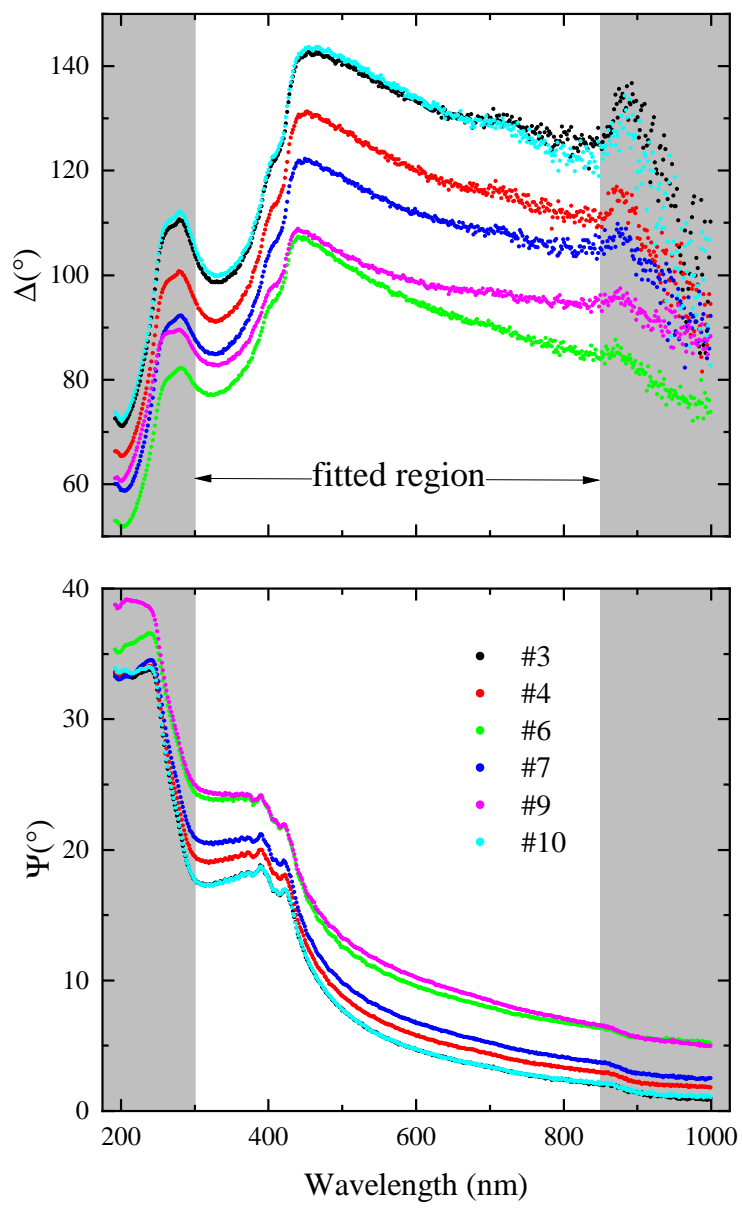

Figure 3: Ellipsometric parameters $\Psi$ and $\Delta$ as a function of wavelength, collected at an angle of incidence of $74^{\circ}$, for different stages of GaAs functionalisation, showing changes on $\mathrm{GaAs}-\mathrm{OH} / \mathrm{COOH}$ SAM (\#3) after incubation with biotin (\#4) and then with neutravidin (\#7). Samples stages are given in Table 1. The increased changes observed in \#6 with respect to \#7 are attributed to non-specific binding, as confirmed by the residual changes measured in \#8 compared to the spectra of stages \#3 and \#10. Spectra collected at additional angles of incidence and corresponding fits are shown in SI Fig. S5 and Fig. S6. 
Table 2: Total layer thicknesses at different stages of functionalisation. Sample stages are given in Table 1. Additional information on the models used for the calculation of thickness is given in SI (Table S1). The values are the average of three measurements in different regions of each sample, with the errors representing the standard deviation.

\begin{tabular}{|c|c|}
\hline Stage & Total thickness, $\mathrm{nm}$ \\
\hline$\# 3$ & $2.31 \pm 0.02$ \\
\hline$\# 4$ & $4.53 \pm 0.17$ \\
\hline$\# 6$ & $7.70 \pm 0.17$ \\
\hline$\# 7$ & $6.41 \pm 0.17$ \\
\hline$\# 9$ & $7.31 \pm 0.65$ \\
\hline$\# 10$ & $2.21 \pm 0.03$ \\
\hline
\end{tabular}

\section{Non-specific binding}

A higher thickness (increase of $1.3 \mathrm{~nm}$ ) was observed for the samples where neutravidin was diluted in PBS (\#6), compared to when diluted in $\operatorname{PBST}(\# 7)$, confirming a larger protein coverage. This is in agreement with the wettability results. In order to clarify if the smaller thickness and hydrophilicity detected for the samples with PBST-diluted neutravidin (\#7) was due to a reduced attachment of neutravidin to biotin or to a decrease in non-specific binding (i.e. the protein adsorption on random surface locations due to electrostatic interactions), we investigated the adsorption of the protein directly onto the $\mathrm{GaAs}-\mathrm{OH} / \mathrm{COOH}$ SAM. The ellipsometry properties of GaAs-M samples incubated with neutravidin diluted in different buffers were measured. Here, a change in $\Delta$ and $\Psi$ is not expected unless the protein binds non-specifically to the SAM, as no biotin is present. The spectra of $\Delta$ and $\Psi$ of stage \#9 (Fig. 3 ) showed differences with respect to the GaAs-M (\#3), despite the PBS/PBST washes, suggesting a change in thickness caused by non-specific binding of the protein. However, when neutravidin was diluted in PBST (\#10) - instead of PBS - followed by PBS/PBST washes, the obtained $\Psi$ and $\Delta$ curves were similar to the ones of stage \#3, indicating an absence of non-specific binding of the protein. These results were further investigated by XPS.

\section{X-ray Photoelectron Spectroscopy (XPS)}

XPS analysis provided further evidence of functionalisation by providing a chemical signature of the outmost surface layer. Here we present high resolution narrow region scans acquired for two key elements, nitrogen and carbon. These elements are present in all the molecules here used for the functionalisation of GaAs except the thiols, which don't incorporate nitrogen.

High resolution scans for the $\mathrm{N}$ 1s region for different stages of functionalisation are shown in Fig. 4. This region is overlapped by $\mathrm{Ga} \mathrm{L}_{2} \mathrm{M}_{45} \mathrm{M}_{45}$ (Ga LMM) Auger peaks, ${ }^{49}$ which complicates quantification of nitrogen species. Nitrogen is absent on the GaAs-M surface (Fig. 4a), as expected, since this element is not present in the thiol molecules (Fig. S1). Biotin attachment is demonstrated by a small shoulder present around $400 \mathrm{eV}$ (Fig. 4b), assigned to $\mathrm{C}-\mathrm{N}$ bonds . ${ }^{50}$ The amount of nitrogen increases after protein attachment (Fig. 4c), as expected, due to the amino acids of the protein. The Ga LMM component overlapping the $\mathrm{N} 1 \mathrm{~s}$ signal is very sensitive to oxidation of the samples, which is also reflected in Ga 2p signals (SI Fig. S10). We attribute the higher level of oxidation observed for some of the sam-

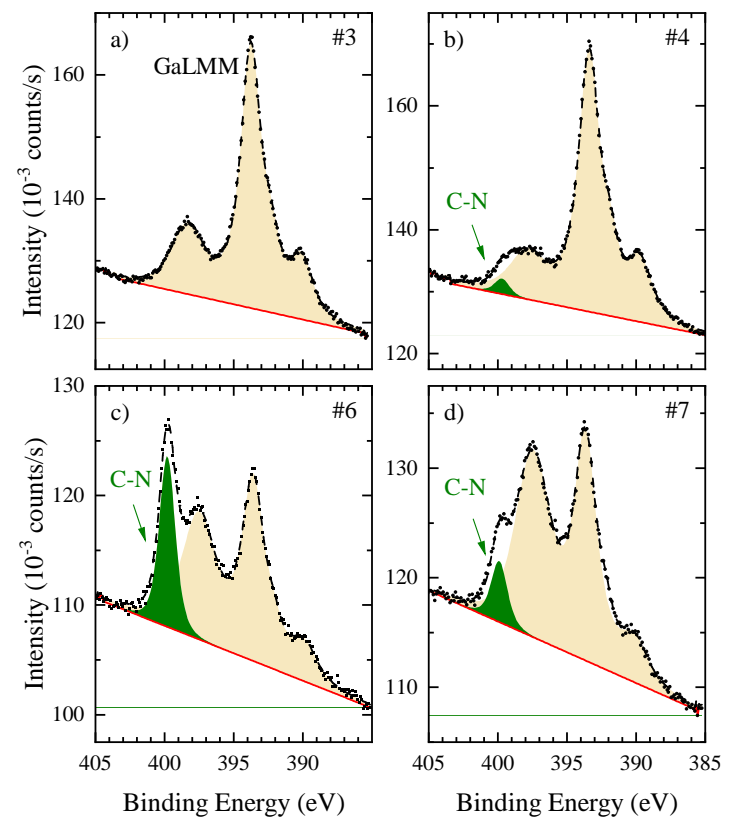

Figure 4: XPS high resolution scans for $\mathrm{N}$ 1s region for samples at different point in the functionalisation as indicated by the sample label. The fitted $\mathrm{N} 1 \mathrm{~s}$ peak at $399.9 \mathrm{eV}$ is shown in green, while the remaining peaks are assigned to the overlapping Ga LMM (in light brown). The background baseline is shown in red.

ples, specifically the samples with neutravidin (Fig. 4c), to a longer exposure to air prior to loading the samples into the spectrometer. Ratios of the areas of the $\mathrm{N} 1 \mathrm{~s}$ to $\mathrm{Ga}$ $2 \mathrm{p}_{3 / 2}$ peaks, calculated from XPS high resolution scans, are shown in Table 3. An increase of $\mathrm{N} 1 \mathrm{~s}$ to $\mathrm{Ga} 2 \mathrm{p}_{3 / 2}$ ratio was observed after biotin attachment and then again after incubation with neutravidin, as expected. The observed increase factor of 1.9 between stage \#4 and \#7 suggests that the ratio of neutravidin to biotin on the surface is approximately 1:25, assuming that each neutravidin molecule has $\sim 100$ atoms of nitrogen, comparing to 4 nitrogen atoms per biotin-containing molecule. This estimation assumes that the $\mathrm{x}$-ray absorption from the protein layer is negligible and the $\mathrm{N}$ signal comes in equal strength from the biotin and the neutravidin layers. This approximation is reasonable considering that the absolute intensity of the Ga peaks measured in stage \#4 and \#7 are similar (within 20\%). The estimated ratio of neutravidin to biotin on the surface is within the expected range, taking into consideration the difference in size for the two molecules. The estimated mass ratio is 246 , considering a mass of 244 Da for the biotin molecule and of $60 \mathrm{kDa}$ for neutravidin. If this value is then converted to an area ratio, by simply considering sphere shape for these molecules, the estimated value is of 39 . Given the simplicity of this calculation - considering spheres of equal density and similar packing density - a variation of $36 \%$ is plausible.

High resolution scans for the $\mathrm{C} 1 \mathrm{~s}$ region for different stages of functionalisation are shown in Fig. 5. This $\mathrm{C} 1 \mathrm{~s}$ signal is superimposed on the high energy tail of the As LMM peak. ${ }^{51}$ As expected, no carbon was detected after in situ argon sputter cleaning applied to the GaAs surface (Fig. S7). For a GaAs sample exposed to the atmosphere, carbon was detected as a result of surface contami- 
Table 3: Ratio of $\mathrm{N}$ 1s and $\mathrm{C}$ 1s peak areas relative to Ga $2 \mathrm{p}_{3 / 2}$, and $\mathrm{C} 1 \mathrm{~s}$ relative component ratios with $\mathrm{C}_{1}$ detected at $284.8 \mathrm{eV}$ (assigned to $\mathrm{C}-\mathrm{C}, \mathrm{C}-\mathrm{H}$ ), $\mathrm{C}_{2}$ detected at $286.2 \mathrm{eV}$ (assigned to $\mathrm{C}-\mathrm{OH}, \mathrm{C}-\mathrm{O}-\mathrm{C}, \mathrm{C}-\mathrm{N}$ ), $\mathrm{C}_{3}$ detected at $288.1 \mathrm{eV}$ (assigned to $\mathrm{C}=\mathrm{O}$ ) and $\mathrm{C}_{4}$ detected at $289.2 \mathrm{eV}$ (assigned to $\mathrm{O}-\mathrm{C}=\mathrm{O}$ ). Where no evident $\mathrm{N}$ 1s (or $\mathrm{C} 1 \mathrm{~s}$ component) peak was present, an upper limit for the ratio was estimated by fitting a peak to the residual; these values are shown in brackets. Additional peak fitting details are shown in Table S2.

\begin{tabular}{|c|c|c|c|c|c|c|}
\hline \multirow{2}{*}{ Stage } & \multirow{2}{*}{$\frac{\mathrm{N} 1 s}{\mathrm{Ga} 2 p_{3 / 2}}$} & \multirow{2}{*}{$\frac{\mathrm{C} 1 s}{\mathrm{Ga} 2 p_{3 / 2}}$} & \multicolumn{4}{|c|}{$\begin{array}{c}\mathrm{C} 1 s \\
\text { components }\end{array}$} \\
\hline & & & $\mathrm{C}_{1}$ & $\mathrm{C}_{2}$ & $\mathrm{C}_{3}$ & $\mathrm{C}_{4}$ \\
\hline$\# 3$ & $(0.003)$ & 1.5 & 0.75 & 0.18 & $(0.01)$ & 0.06 \\
\hline$\# 4$ & 0.10 & 2.2 & 0.69 & 0.28 & 0.03 & $(0.004)$ \\
\hline$\# 6$ & 0.77 & 5.3 & 0.60 & 0.25 & 0.15 & $(0.001)$ \\
\hline$\# 7$ & 0.19 & 2.7 & 0.63 & 0.28 & 0.07 & 0.02 \\
\hline$\# 8$ & 2.13 & 10.6 & 0.56 & 0.27 & 0.17 & $(0.008)$ \\
\hline$\# 9$ & 0.68 & 8.0 & 0.50 & 0.39 & 0.09 & 0.02 \\
\hline$\# 10$ & $(0.002)$ & 1.8 & 0.71 & 0.27 & $(0.004)$ & 0.01 \\
\hline
\end{tabular}

nation ${ }^{52}$ and attributed to $\mathrm{C}-\mathrm{C}$ and $\mathrm{C}-\mathrm{H}$ bonds, positioned at $284.8 \mathrm{eV}$ (see Fig. 5a). Formation of the SAM is noted by additional $\mathrm{C} 1 \mathrm{~s}$ components (Fig. 5b). Specifically the component around $289 \mathrm{eV}$ is assigned to $\mathrm{O}-\mathrm{C}=\mathrm{O}$ bonds, ${ }^{50}$ confirming the presence of carboxyl groups, and the component around $286 \mathrm{eV}$ assigned to $\mathrm{C}-\mathrm{OH}$ and $\mathrm{C}-\mathrm{O}-\mathrm{C}$ bonds, from hydroxyl and ethylene-glycol groups. The concentration of carboxyl groups on the GaAs surface can be tailored by changing the molar fraction of each thiol component in solution (shown in SI, Fig. S8), therefore allowing tailored density of the protein being immobilised on the surface. Scans acquired for the $\mathrm{S} 2 \mathrm{p}$ region (Fig. S9), constitute additional evidence of SAM formation.

The attachment of biotin (Fig. 5c) resulted in a change in the carbon components relative percentages, with an increase in $\mathrm{C}-\mathrm{C}$ and $\mathrm{C}-\mathrm{H}$ peaks, and the appearance of a new component at $288 \mathrm{eV}$, attributed to the carbonyl group $(\mathrm{C}=\mathrm{O} \text { bonds })^{50}$ of the biotin. In addition, an increase of $\mathrm{C}$ 1 s to $\mathrm{Ga} 2 \mathrm{p}_{3 / 2}$ ratio was observed (Table 3 ). The increase factor of 1.5 (between stage \#3 and \#4) suggests that all the carboxyl thiol groups reacted with biotin molecules. This can be explained by the change in the $\mathrm{C}$ content of these films as follows. The dual-component SAM formed on the GaAs surface presents equimolar fractions of each thiol (results not shown, from water contact angle measurements of pure SAMs). The number of $\mathrm{C}$ atoms is 19 per carboxylOEG-thiol, 17 per hydroxyl-OEG-thiol, and 16 per biotin. Therefore, an increase factor of $(19+17+16) /(19+17) \approx 1.44$ is expected for $\mathrm{C}$ if all the carboxyl groups react with biotin via carbodiimide chemistry. Further attachment of the protein (Fig. 5 d) resulted, again, in an increase of $\mathrm{C} 1 \mathrm{~s}$ to $\mathrm{Ga}$ $2 \mathrm{p}_{3 / 2}$ ratio and a change in the relative percentages of carbon components.

\section{Non-specific binding}

Fig. 6 shows how the amount of protein adsorbed onto the GaAs-M surface varied as a function of the protein and washing buffers. The prominent nitrogen peak in Fig. 6a confirms that the protein, when diluted in PBS, adsorbed onto the SAM, even in the absence of the specific binding site of the biotin. A smaller but still evident peak was observed when the samples were rinsed with PBST after incubation with

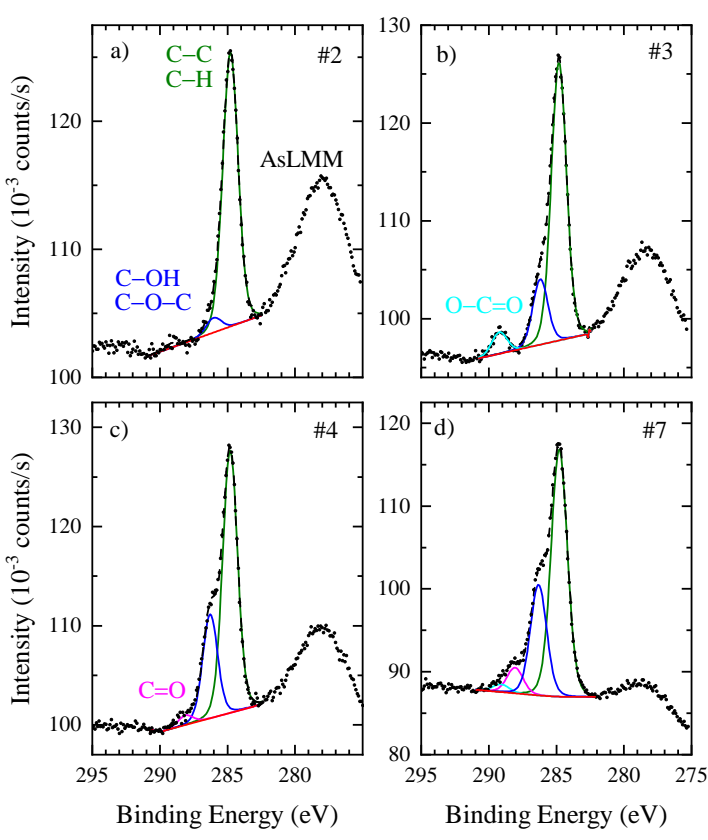

Figure 5: XPS high resolution spectra for $\mathrm{C}$ 1s region with fitted components (symbols). This region is overlapped by As LMM auger peaks (not fitted). a) was acquired from a sample of GaAs that had been exposed to air. b-d) are representative scans for different stages of functionalisation of the GaAs samples, as indicated by the sample label. The lines are the fit of the $\mathrm{C}-\mathrm{C} / \mathrm{C}-\mathrm{H}$ (green), $\mathrm{C}-\mathrm{OH} / \mathrm{C}-\mathrm{O}-\mathrm{C}$ (blue), $\mathrm{C}=\mathrm{O}$ (magenta) and $\mathrm{O}-\mathrm{C}=\mathrm{O}$ (cyan), respectively, with the dashed black line their sum. The background baseline is shown in red.

the protein diluted in PBS (Fig. 6b). In contrast, when the protein was diluted in PBST and the samples were rinsed with PBS/PBST (Fig. 6c), no nitrogen peak was detected, indicating that no protein adsorbed onto the surface. These results are in agreement with the contact angle and ellipsometry observations, demonstrating the absence of non-specific binding when the protein is diluted in PBST followed by PBS/PBST rinse. This conclusion is also confirmed by the study of the XPS spectra of the C 1s peak (see Fig. S11).

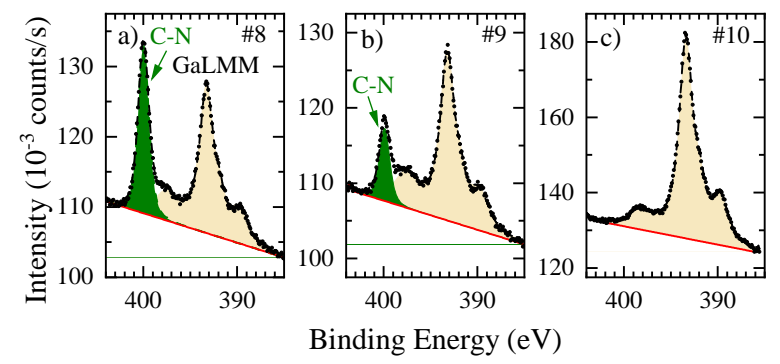

Figure 6: Same as Fig. 4 for samples of GaAs-M incubated with neutravidin as a function of the protein dilution and washing buffers, as indicated by the sample label. 


\section{Conclusions}

Systematic studies on the biofunctionalisation of GaAs are scarce, restraining the opportunity to take advantage of its unique optical and electrical properties for biosensing. We have demonstrated biofunctionalisation of GaAs (100) with a biotin ligand and its biological counterpart, neutravidin, based on combined XPS, contact angle and spectroscopic ellipsometry.

The formation of monolayers was confirmed and the total thickness of the film on GaAs after protein attachment was under $10 \mathrm{~nm}$, validating the suitability of this functionalisation strategy for applications that require low thickness of the film formed on the substrate, such as optical biosensors that make use of the evanescent wave of light at the GaAs/water interface. The results show that the biotin molecules reacted efficiently with the carboxyl groups on the surface via carbodiimide crosslinking, providing a coverage of neutravidin:biotin with a molecular ratio of 1:25.

The functionalisation and characterisation strategy herein described can be applied to the immobilisation of other targets on GaAs for biological/biomedical applications. For example, this platform can be used for the immobilisation of biotinylated antibodies or an enzyme or target protein conjugated to biotin ${ }^{3,19}$ Being composed of a dual-component monolayer, this system provides flexibility for customizing the density of binding sites on the surface.

Despite the use of OEG-containing SAMs, we detected significant non-specific adsorption of the protein, when using PBS as dilution buffer. Changing the protein dilution buffer to PBST, followed by PBST thorough rinse, non-specifing binding was eliminated.

\section{Acknowledgements}

This research was supported by the Sêr Cymru II programme (Case ID 80762-CU-148) which is part-funded by Cardiff University and the European Regional Development Fund through the Welsh Government. XPS data collection was performed at the EPSRC National Facility for XPS (HarwellXPS), operated by Cardiff University and UCL, under contract No. PR16195. We thank Oliver Williams and Evan Thomas for providing us training and access to the Spectroscopic Ellipsometer system. We also thank Neal Fairley from CasaXPS for the support provided regarding the use of CasaXPS software for data analysis, and Maria White for preparing samples with varying carboxyl-thiol molar fractions, shown in SI.

\section{References}

(1) Budz, H.; Ali, M.; Li, Y.; Lapierre, R. Photoluminescence model for a hybrid aptamer-GaAs optical biosensor. Journal of Applied Physics 2010, 107.

(2) Lacour, V.; Herth, E.; Lardet-Vieudrin, F.; Dubowski, J.; Leblois, T. GaAs based on bulk acoustic wave sensor for biological molecules detection. Procedia Engineering 2015, 120, 721-726.

(3) Aziziyan, M.; Hassen, W.; Morris, D.; Frost, E.; Dubowski, J. Photonic biosensor based on photocorrosion of GaAs/AlGaAs quantum heterostructures for detection of Legionella pneumophila. Biointerphases 2016, 11, 019301.
(4) Bhalla, N.; Jolly, P.; Formisano, N.; Estrela, P. Introduction to biosensors. Essays in Biochemistry 2016 , $60,1-8$.

(5) Kim, J.; Campbell, A.; de Ávila, B. E.-F.; Wang, J. Wearable biosensors for healthcare monitoring. Nature Biotechnology 2019, 37, 389-406.

(6) Yam, C.; Pradier, C.; Salmain, M.; Marcus, P.; Jaouen, G. Binding of Biotin to Gold Surfaces Functionalized by Self-Assembled Monolayers of Cystamine and Cysteamine: Combined FT-IRRAS and XPS Characterization. Journal of Colloid and Interface Science 2001, 183-189.

(7) Conde, J.; Dias, J.; Grazu, V.; Moros, M.; Baptista, P.; de la Fuente, J. Revisiting 30 years of biofunctionalization and surface chemistry of inorganic nanoparticles for nanomedicine. Frontiers in Chemistry 2014, 2.

(8) Lapin, N.; Seitz, O.; Chabal, Y. Biointerface Characterization by Advanced IR Spectroscopy; Elsevier, 2011; Chapter Infrared analysis of biomolecule attachment to functionalized silicon surfaces, pp 83-113.

(9) Georgakilas, V.; Perman, J. A.; Tucek, J.; Zboril, R. Broad Family of Carbon Nanoallotropes: Classification, Chemistry, and Applications of Fullerenes, Carbon Dots, Nanotubes, Graphene, Nanodiamonds, and Combined Superstructures. Chemical Reviews 2015, 115, 4744-4822.

(10) Contreras-Naranjo, J.; Aguilar, O. Suppressing NonSpecific Binding of Proteins onto Electrode Surfaces in the Development of Electrochemical Immunosensors. Biosensors 2019, 9 .

(11) Love, J.; Estroff, L.; Kriebel, J.; Nuzzo, R.; Whitesides, G. Self-Assembled Monolayers of Thiolates on Metals as a Form of Nanotechnology. Chemical Reviews 2005, 105, 1103-1170.

(12) Laromaine, A.; Mace, C. Organic Nanomaterials: Synthesis, Characterization, and Device Applications; John Wiley \& Sons, Inc., 2013; Chapter SelfAssembled Monolayers as Model Biosurfaces, pp 369395 .

(13) Voznyy, O.; Dubowski, J. Adsorption Kinetics of Hydrogen Sulfide and Thiols on GaAs (001) Surfaces in a Vacuum. The Journal of Physical Chemistry $C$ 2008, 112, 3726-3733.

(14) Sheen, C.; Shi, J.; Martensson, J.; Parikh, A.; Allara, D. A New Class of Organized Self-Assembled Monolayers: Alkane Thiols on GaAs (100). Journal Am. Chem. Soc. 1992, 114, 1515-1517.

(15) Ding, X.; Dubowski, J. Surface passivation of (001) GaAs with self-assembled monolayers of long-chain thiols. Photon Processing in Microelectronics and Photonics IV. 2005.

(16) Huang, X.; Xia, P.; Wang, X.; Hu, Y. Surface passivation of $\mathrm{GaAs}(001)$ by $\mathrm{Hg} 2 \mathrm{Cl} 2$ nanoplates combined with hexadecanethiol. Applied Surface Science 2019, 473, 141-147. 
(17) Lee, K.; Nair, P.; Scott, A.; Alam, M.; Janes, D. Device considerations for development of conductancebased biosensors. Journal of Applied Physics 2009, 105, 102046 .

(18) Cho, Y.; Ivanisevic, A. Covalent Attachment of TAT Peptides and Thiolated Alkyl Molecules on GaAs Surfaces. The Journal of Physical Chemistry B 2005, 109, 12731-12737.

(19) Nazemi, E.; Hassen, W.; Frost, E.; Dubowski, J. Growth of Escherichia coli on the GaAs (001) surface. Talanta 2018, 178, 69-77.

(20) Duplan, V.; Miron, Y.; Frost, E.; Grandbois, M.; Dubowski, J. Specific immobilization of influenza A virus on GaAs (001) surface. Journal of Biomedical Optics 2009, 14, 054042 .

(21) Bienaime, A.; Leblois, T.; Gremaud, N.; Chaudon, M.J.; Osta, M.; Pecqueur, D.; Ducoroy, P.; Elie-Caille, C. Influence of a Thiolate Chemical Layer on GaAs (100) Biofunctionalization: An Original Approach Coupling Atomic Force Microscopy and Mass Spectrometry Methods. Materials 2013, 6, 4946-4966.

(22) McGuiness, C.; Shaporenko, A.; Mars, C.; Uppili, S.; Zharnikov, M.; Allara, D. Molecular Self-Assembly at Bare Semiconductor Surfaces: Preparation and Characterization of Highly Organized Octadecanethiolate Monolayers on GaAs(001). Journal of the American Chemical Society 2006, 128, 5231-5243.

(23) McGuiness, C.; Shaporenko, A.; Zharnikov, M.; Walker, A.; Allara, D. Molecular Self-Assembly at Bare Semiconductor Surfaces: Investigation of the Chemical and Electronic Properties of the AlkanethiolateGaAs(001) Interface. The Journal of Physical Chemistry $C$ 2007, 111, 4226-4234.

(24) Mancheno-Posso, P.; Muscat, A. Self-assembly of alkanethiolates directs sulfur bonding with $\operatorname{GaAs}(100)$. $A p$ plied Surface Science 2017, 397, 1-12.

(25) Lacour, V.; Moumanis, K.; Hassen, W.; Elie-Caille, C.; Leblois, T.; Dubowski, J. Formation Kinetics of Mixed Self-Assembled Monolayers of Alkanethiols on GaAs(100). Langmuir 2017, 35, 4415-4427.

(26) Jun, Y.; Zhu, X.; Hsu, J. Formation of Alkanethiol and Alkanedithiol Monolayers on GaAs(001). Langmuir 2006, 22, 3627-3632.

(27) Voznyy, O.; Dubowski, J. Structure of thiol selfassembled monolayers commensurate with the GaAs (001) surface. Langmuir 2008, 24, 13299-13305.

(28) Hermanson, G. (Strept)avidin-Biotin Systems. Bioconjugate Techniques. 2013

(29) Bayer, E.; Wilchek, M. The Use of the Avidin-Biotin Complex as a Tool in Molecular Biology. Methods of Biochemical Analysis 1980, 26.
(30) Vermette, P.; Gengenbach, T.; Divisekera, U.; Kambouris, P.; Griesser, H.; Meagher, L. Immobilization and surface characterization of NeutrAvidin biotinbinding protein on different hydrogel interlayers. Journal of Colloid and Interface Science 2003, 259, 13-26.

(31) Prime, K. L.; Whitesides, G. M. Adsorption of Proteins onto Surfaces Containing End-Attached Oligo(ethylene oxide): A Model System Using SelfAssembled Monolayers. Journal of the American Chemical Society 1993, 115, 10714-10721.

(32) Wang, R.; Kreuzer, H.; Grunze, M. Molecular conformation and solvation of oligo(ethylene glycol)terminated self-assembled monolayers and their resistance to protein adsorption. Journal of Physical Chemistry B 1997, 101, 9767-9773.

(33) Zheng, J.; Li, L.; Tsao, H.; Sheng, Y.; Chen, S.; Jiang, S. Strong repulsive forces between protein and oligo (ethylene glycol) self-assembled monolayers: A molecular simulation study. Biophysical Journal 2005, 89, 158-166.

(34) Wang, F.; Anderson, M.; Bernards, M.; Hunt, H. PEG functionalization of whispering gallery mode optical microresonator biosensors to minimize non-specific adsorption during targeted, label-free sensing. Sensors (Switzerland) 2015, 15, 18040-18060.

(35) Harder, P.; Grunze, M.; Dahint, R.; Whitesides, G.; Laibinis, P. Molecular conformation in oligo(ethylene glycol)-terminated self-assembled monolayers on gold and silver surfaces determines their ability to resist protein adsorption. Journal of Physical Chemistry B 1998, 102, 426-436.

(36) Chatterjee, J. Limiting conditions for applying the spherical section assumption in contact angle estimation. Journal of Colloid and Interface Science 2003, 259, 139-147.

(37) Fairley, N. CasaXPS Manual 2.3.15 Rev1.2. 2009.

(38) Major, G.; Shah, D.; Fernandez, V.; Fairley, N.; Linford, M. Advanced Line Shapes in X-Ray Photoelectron Spectroscopy I. The Asymmetric Lorentzian (LA) Line Shape. Vacuum Technology $\&$ Coating 2020,

(39) Demanet, C.; Marais, M. A Multilayer Model for GaAs Oxides Formed at Room Temperature in Air as Deduced from an XPS Analysis. Surface and Interface Analysis 1985, 7 .

(40) Baca, A.; Ashby, C. Cleaning and passivation. Fabrication of GaAs devices. 2005.

(41) Bain, C.; Whitesides, G. Modeling organic surfaces with self-assembled monolayers. Advanced Materials 1989, 1, 110-116.

(42) Wang, H.; Chen, S.; Li, L.; Jiang, S. Improved Method for the Preparation of Carboxylic Acid and Amine Terminated Self-Assembled Monolayers of Alkanethiolates. Langmuir 2005, 21, 2633-2636. 
(43) Myrskog, A.; Anderson, H.; Aastrup, T.; Ingemarsson, B.; Liedberg, B. Esterification of Self-Assembled Carboxylic-Acid-Terminated Thiol Monolayers in Acid Environment: A Time-Dependent Study. Langmuir 2010, 26, 821-829.

(44) Pale-Grosdemange, C.; Simon, E.; Prime, K.; Whitesides, G. Formation of Self-Assembled Monolayers by Chemisorption of Derivatives of Oligo(ethylene glycol) of Structure $\mathrm{HS}(\mathrm{CH} 2) 11(\mathrm{OCH} 2 \mathrm{CH} 2) \mathrm{mOH}$ on Gold. Journal of the American Chemical Society 1991, 113, $12-20$.

(45) Lee, S. W.; Laibinis, P. E. Protein-resistant coatings for glass and metal oxide surfaces derived from oligo(ethylene glycol)-terminated alkyltrichlorosilanes. Biomaterials 1998, 19, 1669-1675.

(46) Castner, D. Biomedical surface analysis: Evolution and future directions (Review). Biointerphases 2017, 12, $02 \mathrm{C} 301$.

(47) Rios, F.; Smirnov, S. Biochemically Responsive Smart Surface. ACS Applied Materials and Interfaces 2010, 1, $768-774$.

(48) Tompkins, H. A User's Guide to Ellipsometry; Academic Press, Inc., 1993.

(49) Antonides, E.; Janse, E.; Sawatzky, G. LMM Auger spectra of $\mathrm{Cu}, \mathrm{Zn}, \mathrm{Ga}$ and Ge. Transition probabilities, term splittings and effective Coulomb interaction. Physical Review B 1977, 15, 1669-1679.

(50) Wagner, C.; Riggs, W.; Davis, L.; Moulder, J. In Handbook of X-ray Photoelectron Spectroscopy; Muilenberg, G., Ed.; Perkin-Elmer Corp. Physical Electronics Division, 1979.

(51) Roberts, E.; Weightman, P.; Johnson, C. Photoelectron and L2,3 MM Auger electron energies for arsenic. Journal of Physics C: Solid State Physics 1975, 8, 1301-1309.

(52) Barr, T.; Seal, S. Nature of the use of adventitious carbon as a binding energy standard. Journal of Vacuum Science $\&$ Technology A: Vacuum, Surfaces, and Films 1995, 13, 1239-1246. 